\title{
Design and Implementation of Wirelessly Controlled Mobile Robot for Navigation of Multi Locations
}

\author{
${ }^{1}$ Karrar AlSlmman, ${ }^{2}$ Hayder Hasan Ali and ${ }^{1}$ Zain-Aldeen S.A. Rahman \\ ${ }^{1}$ Department of Electrical Techniques, Qurna Technical Institute, \\ Southern Technical University, Qurna, Basra, Iraq \\ ${ }^{2}$ Department of Electronics Techniques, Basra Technical Institute, \\ Southern Technical University, Basra, Iraq
}

\begin{abstract}
Recently, mobile robots are widely used to perform difficult tasks in many applications as industrial manufacturing, dangerous areas and military, medical industry and for assisting the hampered human. This study focused on the interface between the human and machine. In this project, we designed and developed a wirelessly controlled mobile robot. The robot navigation is controlled by using a Graphical User Interface (GUI) through a wireless protocol with help of a computer (PC) by using a LabVIEW Software. We can represented the whole project by a system which consists of two parts, first part is a controller part that presented by the $\mathrm{PC}$ and second part is the mobile robot. The mobile robot work as a slave to a coordinator in the other hand the PC works as a coordinator. A microcontroller presented by an Arduino Uno microcontroller type and $\mathrm{HC}-12$ wireless serial communication mote achieve the wireless communication between the robot and the PC. Steppers motors with its derivers (A4988 stepper motor derive) are used for implementation of this mobile robot and the motion of this robot is controlled through a series of pulses. This robot can navigate in many paths; in this project, three different paths are navigated. Depending on the signal received from the PC the navigation of robot reached the determined location. Wireless control of mobile robot reach many benefits including the using of robot in dangerous application and avoidance wires or cables that disrupt the motion of the robot.
\end{abstract}

Key words: Mobile robot, control, wireless, system, GUI, PC

\section{INTRODUCTION}

With the growth of technologies and science, the mobile robots are playing more significant roles in many fields, including industrial automation, medical service, dangerous field applications and household services. A mobile robot principally can be defined is a machine that has the ability for moving in depending on a certain instructions (Borker et al., 2014).

Numerous robotics researchers, search for improving the control systems for mobile robot motion and its tasks. This improvement in the control system for doing more functions these functions upcoming together. Many projects and searches in the word focused on machinery of the interface between the human and machines. This interface can be reached by different technologies depending on the application (Patil et al., 2016). A wireless technology can be used for controlling the mobile robot motion and its tasks. Many wireless technologies can be used such as Wi-Fi, Bluetooth and wireless LAN (Kahar et al., 2012). By Lee et al. (2014) introduced a mobile robot controlled by smartphone based on iOS. The suggested scheme is consist of a smartphone and a mobile. Wi-Fi communication board is used in the mobile robot for implementing the wireless communication between the smartphone and the robot. There are two control methodologies are provided for the suggested control system including a desired touch board control and the smartphone control. These two topologies can be used to drive the mobile robot. By Li et al. (2015) developed remote-controlled mobile robot for environment monitoring. In this research, an NRF24L01 wireless communication motes is used for reaching the data communication between the host $\mathrm{PC}$ and the mobile robot. Therefore, there are a real time monitoring for the robot motion. In the other hand, the data required from the mobile robot send wirelessly to the $\mathrm{PC}$ in serial form via. NRF24L01 wireless modules. Then the PC send the

Corresponding Author: Karrar AlSlmman, Department of Electrical Techniques, Qurna Technical Institute, Southern Technical University, Qurna, Basra, Iraq 
determined instructions to the fixed controller in the mobile robot through the NRF24L01 wireless modules. By Teodorescu et al. (2016) presented the use of wireless technology in the controlling of the mobile robot. In this project, the control of mobile robot is achieved wirelessly by using a PC through a GUI deigned by the LabVIEW Software. They used the Zigbee technology for the wireless communication between the $\mathrm{PC}$ and the mobile robot. By Chen et al. (2017) designed and implemented a remotely control system for nuclear pollution disposal robot. The wirelessly control of this system designed based on the TCP/IP protocol and user server mode. The remote host and mobile robot is realized by Winsock technology. The Visual $\mathrm{C}++$ programing language is used for writing the remote control system software then that is coded by a modular design method and structured in a controller box. By Farkhana and Hanan (2008), introduced a study focused the mobile robot control based on wireless technologies. Generally, this study aims to discuss and analyses the Mobile ad Hoc Network (MANET) technology that applicable in the control of the mobile robot. As well as the review, the researchers practically tested a prototype mobile robot based on the MANET for enabling the reader to understand the advantages and disadvantages of the MANET.

Here, the control of the mobile robot is realized remotely by a PC through a GUI designed by the LabVIEW Software and can be used for robot control and robot movement tracking.

\section{MATERIALS AND METHODS}

The system structure: In this project, a system of a prototype mobile robot is controlled remotely designed and implemented. For implementing the wireless control system, we can describe the wireless protocol as following. The mobile robot will work as a slave and must communicate with a central unit that presented by the PC which is considered a control coordinator unit. Figure 1 illustrates the proposed block diagram of the wireless control the mobile robot control through the PC. Generally, for controlling the drive of the mobile robot and its directions, initially we must control the speed and the direction of the rotation of the mobile robot drive motor. In this project, the $\mathrm{PC}$ determine the goals (set points) of the mobile robot. The mobile robot respond to the control unit and provided it by a feedback signal.

The PC-control unit: The control unit plays a key role in the designed control system of the mobile rbot. The

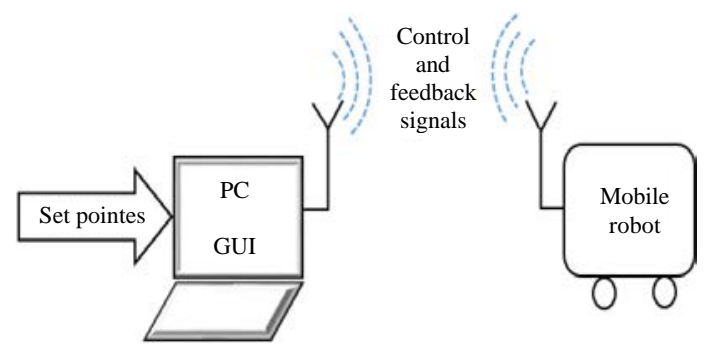

Fig. 1: The system structure

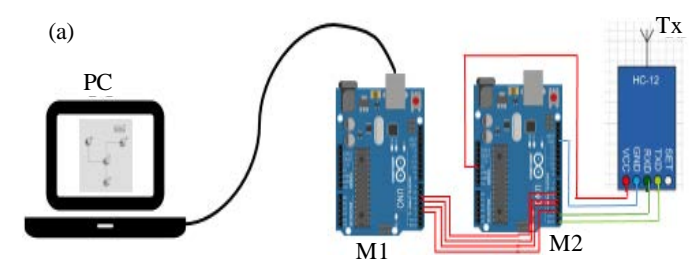

(b)

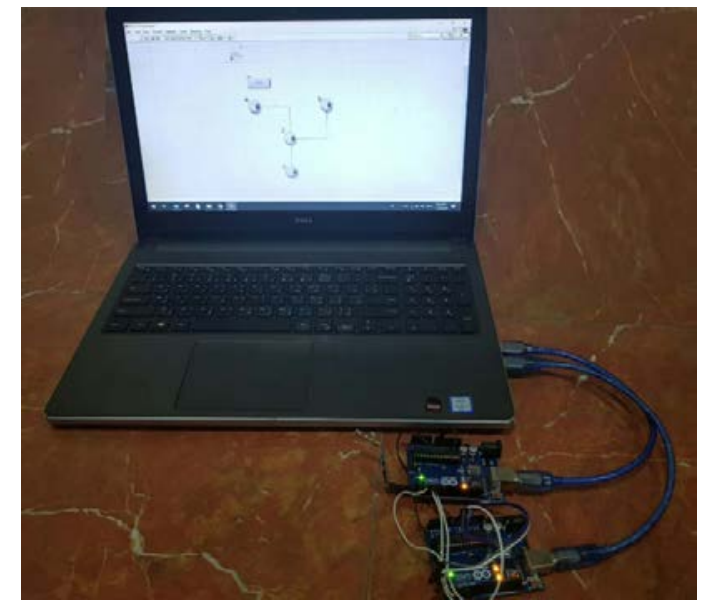

Fig. 2: PC-control unit; a) Schematic diagram and b) Hardware construction

hardware of control unit consists of the flowing devices: a $\mathrm{PC}$ which used for controlling and monitoring the robot navigation, two Arduino Uno boards ( $\mathrm{M} 1$ and $\mathrm{M} 2$ ), one for interfacing with the $\mathrm{PC}$ and the other for connecting with the $\mathrm{HC}-12$ wireless module and the $\mathrm{HC}-12$ wireless module allows the wireless communication between the $\mathrm{PC}$ and the mobile robot. Figure 2a, shows the PC schematic diagram and Fig. 2b, shows the construction of a prototype $\mathrm{PC}$ control unit that used for implementing the system. Table 1 shows the pins connections of the PC-control unit devices (Table 2).

The mobile robot: The hardware of the mobile robot consisted of an Arduino Uno board (M3) and the feedback sensors, two servomotors, motor drive, two 
Table 1: The PC-control unit pin connections

\begin{tabular}{lcll}
\hline \multicolumn{3}{c}{ Pins symbols } \\
\hline M1 & M2 & M2 & Tx \\
\hline 2 & 2 & $0(\mathrm{Rx})$ & Tx \\
3 & 3 & $1(\mathrm{Tx})$ & $\mathrm{Rx}$ \\
4 & 4 & $+5 \mathrm{v}$ & VCC \\
5 & 5 & GND & GND \\
\hline
\end{tabular}

Table 2: The mobile robot pins connections.

\begin{tabular}{lllll}
\hline \multicolumn{5}{c}{ Pin symbols } \\
\hdashline $\mathrm{Rx}$ & $\mathrm{M} 3$ & $\mathrm{M} 3$ & Stepper drive (R) & Stepper drive (L) \\
$\mathrm{Tx}$ & $\mathrm{Rx}$ & 2 & Step & \\
$\mathrm{RX}$ & $\mathrm{Tx}$ & 5 & Dir. & Step \\
VCC & $+5 \mathrm{~V}$ & 3 & & Dir. \\
GND & GND & 6 & & ENB \\
& & 8 & ENB & GND \\
\hline
\end{tabular}

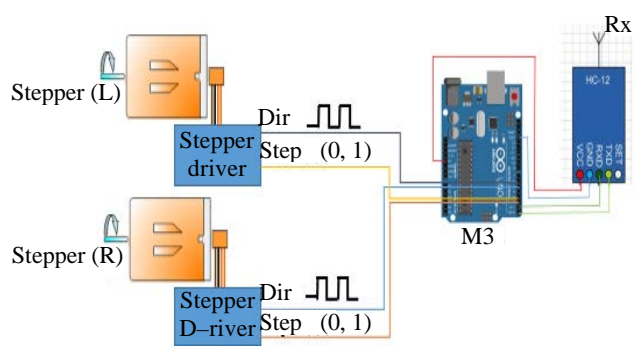

Fig. 3: The mobile robot schematic diagram

$\mathrm{H}$-bridge, HC-12 wireless module and $+9 \mathrm{~V}$ batteries these component are set up and connected as shown in Fig. 3. The front and back view of the hardware construction of a prototype mobile robot are shown in Fig. 4a and b, respectively.

The Arduino Uno board is the major unit in the mobile robot and its present the brain of the mobile robot. It's receive the signals wirelessly from the $\mathrm{PC}$ control unit through the HC-12 wireless module and process these signals and then acknowledge a determined signals to the servomotors through the motor derive. The motor drive and H-bridge are connected to the Arduino Uno board and the signals acknowledged from the Arduino Uno board are used for deriving the servomotors. Therefore, the movement and the rotation of the servomotors motor depends on the transmitted signals from PC control unit. The two H-bridge are responding for the movement and the direction of the servomotors thought the motor drive signals. Figure 5 demonstrates the block diagrams of the movement, the right and left directions of the mobile robot. The pins connections of the mobile robot illustrated in Table 2.

The software description: The software part plays a key role in the aimed system, its contain the software
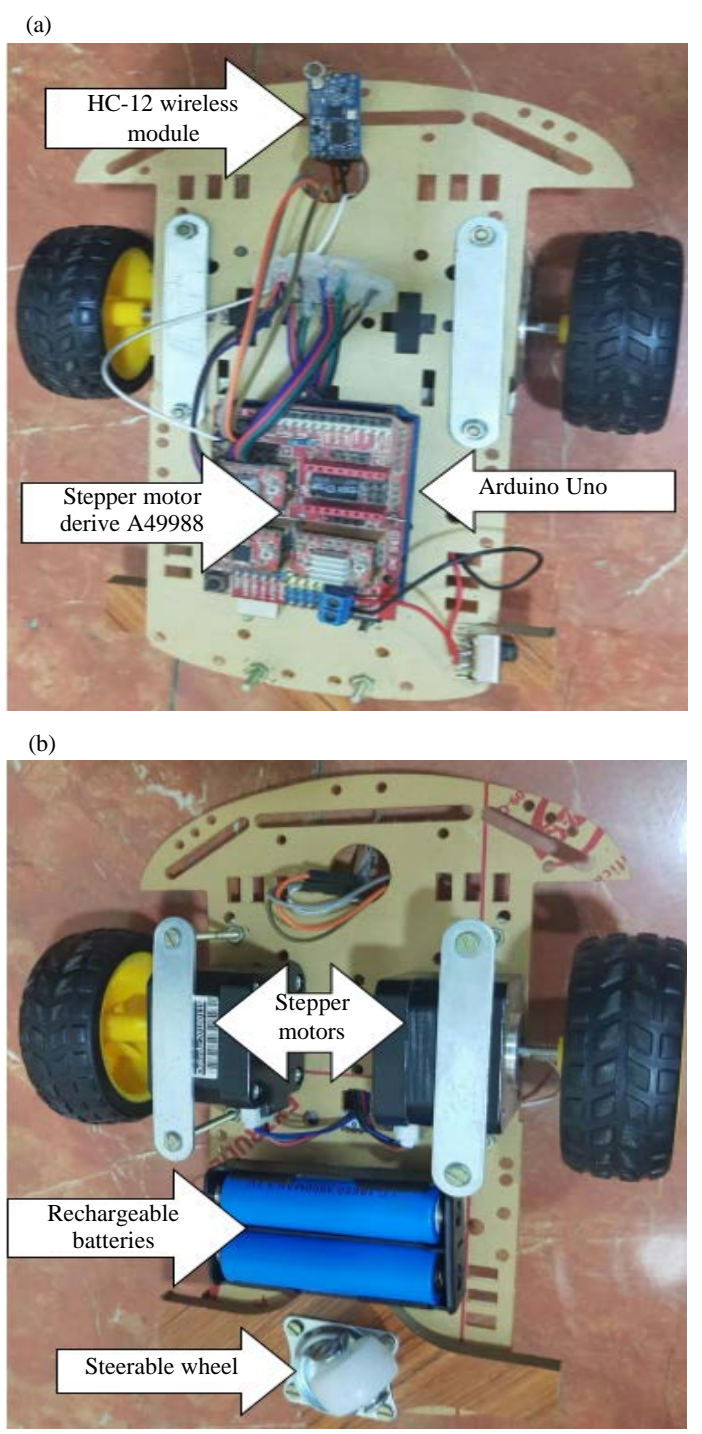

Fig. 4: Hardware structure of the mobile robot; a) Front view and b) Back view
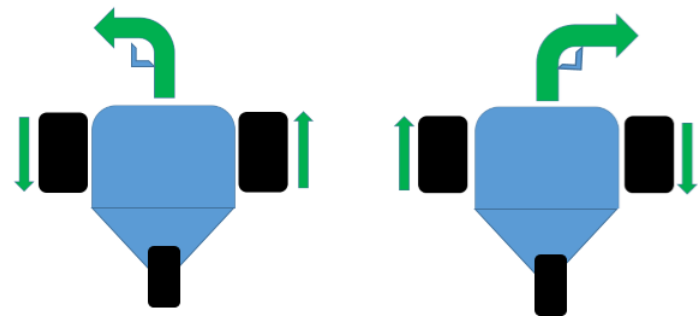

Fig. 5: The mobile robot directions

programs that used for developing the system and the system protocol. The software programs that used in this project include, Arduino Integrated Development Environment (IDE) and the LabVIEW. The Arduino IDE 


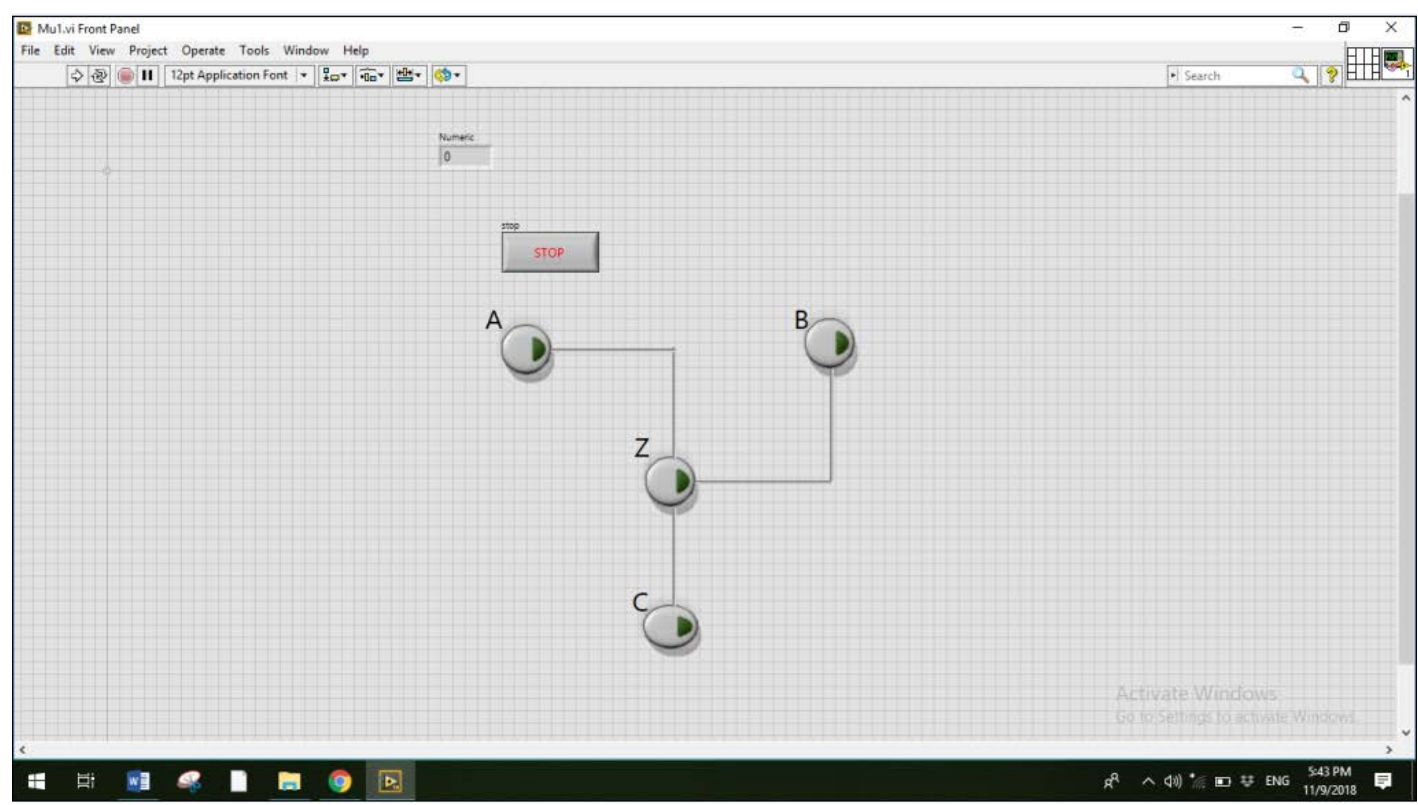

Fig. 6: LabVIEW GUI

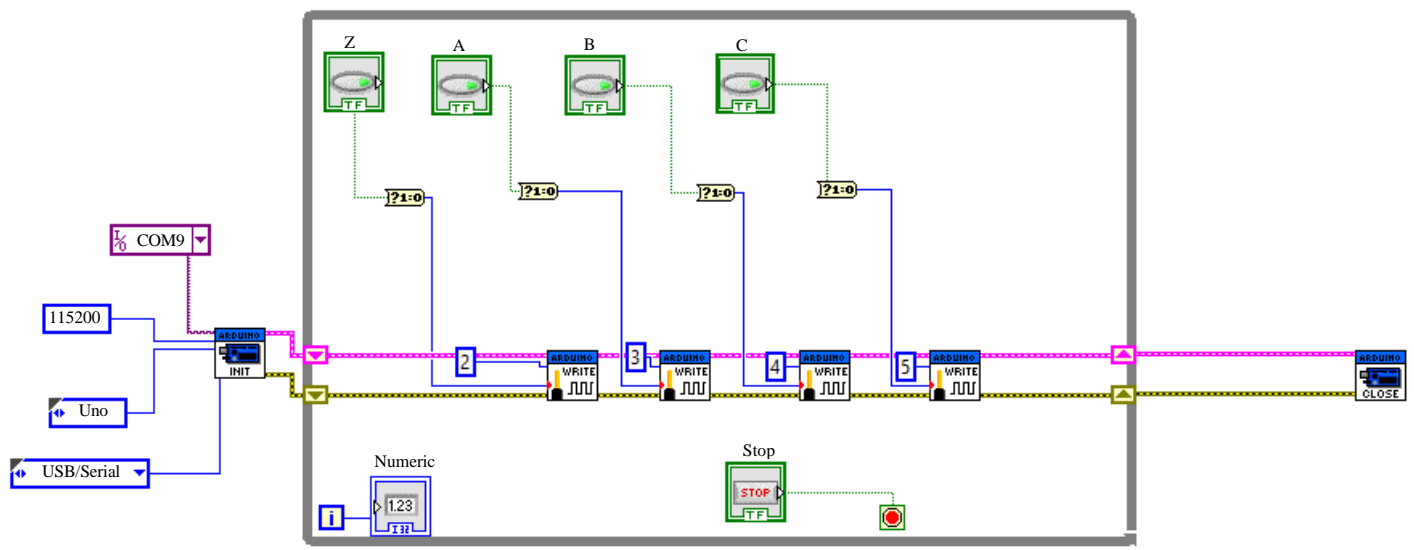

Fig. 7: LabVIEW schematic diagram

Software that employs C language used for programming the Arduino Uno board which contents the microcontroller. The LabVIEW Software is used for designing the GUI in the PC-control unit that allows the interface between the user and the PC to control the mobile robot. In addition, the LabVIEW Software achieves the interface between the PC and the Arduin Uno board. GUI and LabVIEW schematic diagram are presented in Fig. 6 and 7, respectively. In the designed GUI the $Z$ loacation presents the reference location, in the other hand the locations A-C present the mobile robot goals. The navigation paths of the robot are illustrated in the GUI presented by the lines.
The system protocol: As we said above, $\mathrm{PC}$, stepper motors are connected to the Arduino Uno and HC-12 wireless module are used for developing the system. The wireless protocol between the PC control unit and the mobile robot can be described as following, the $\mathrm{PC}$ control unit send a determined instruction signals wirelessly to the robot and the robot receives these instruction signals from PC control unit, the control unit in the robot presented by the Arduino Uno board served as input signal to the controller and then the stepper motors operate according to the controller control signal. In the other hand, the robot must also provide a feedback signals to the PC control unit display in the designs GUI, 


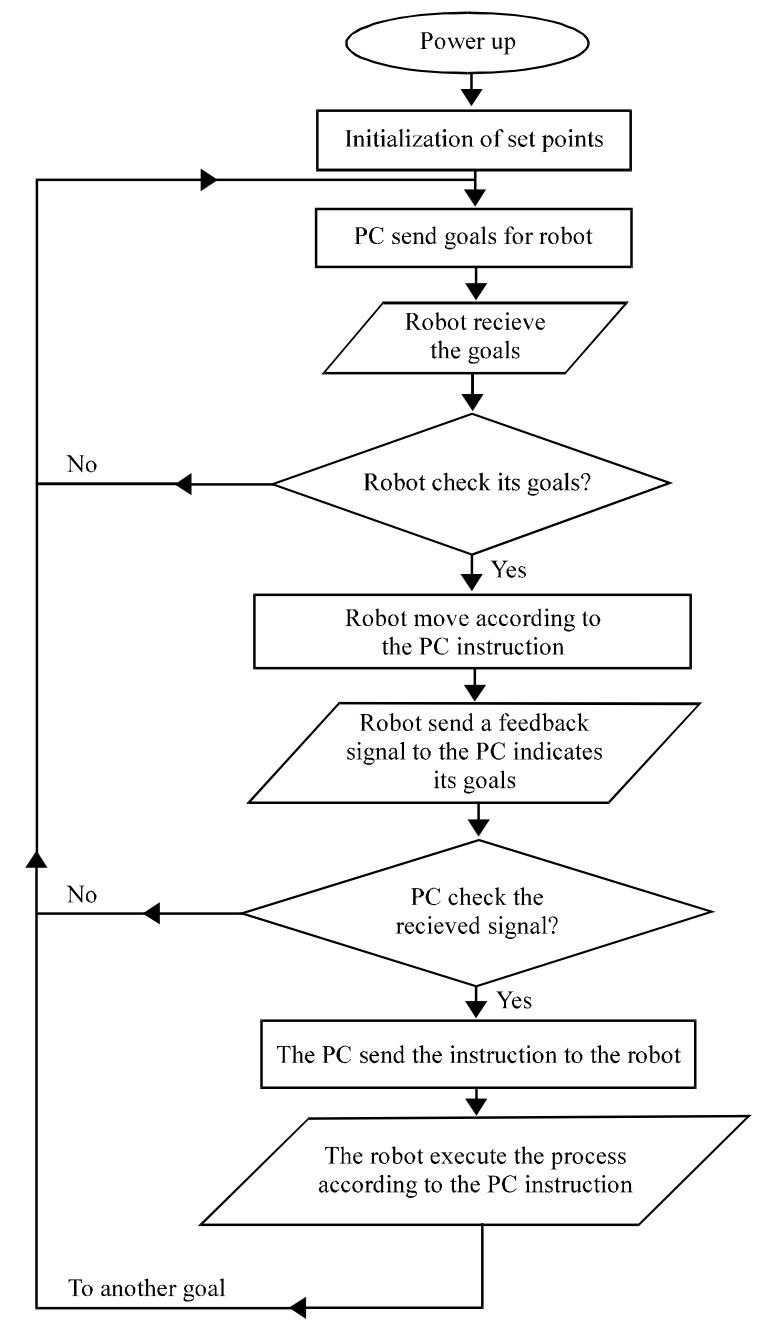

Fig. 8: The flow charts of the system protocol

so, the user note the robot response to the specified instruction. This wireless controlling of the mobile robot not only control the robot motion but also will do the determined tasks. The flow chart that describes the system protocol is shown in Fig. 8.

\section{RESULTS AND DISCUSSION}

Our proposed system of the mobile robot is designed and it has been successfully employed as a prototype model. This prototype was tested and it has presented a good performance. The steps of algorithm (A) summarizes how the PC-control unit derive the navigation of the mobile robot through the GUI with its determined paths dimensions that shown in Fig. 9 where $\mathrm{a} 1, \mathrm{a} 2, \mathrm{~b} 1, \mathrm{~b} 2$ and $\mathrm{c}$ is the paths dimensions that determine by the designer and the

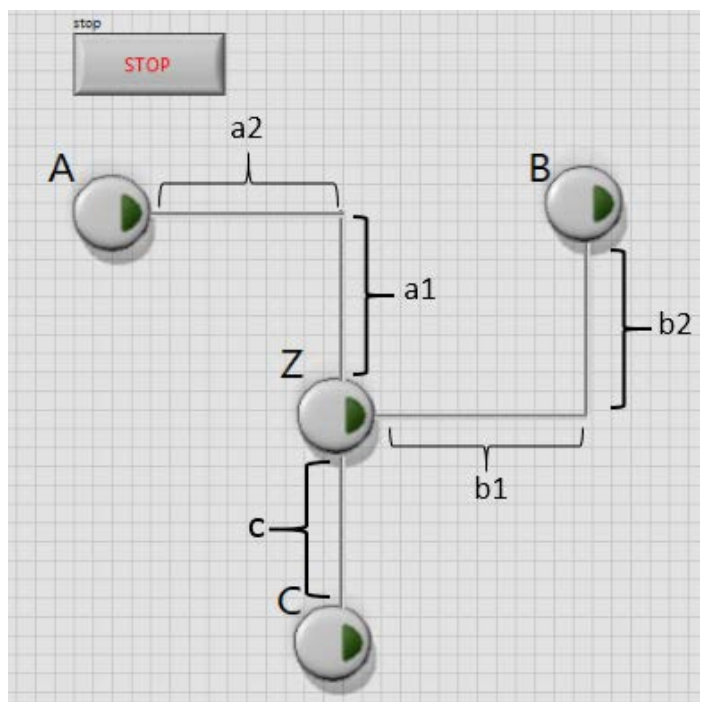

Fig. 9: GUI with paths dimensions

steps of the algorithm (B) describes how the mobile robot responds to the control signals received from the PC-control unit.

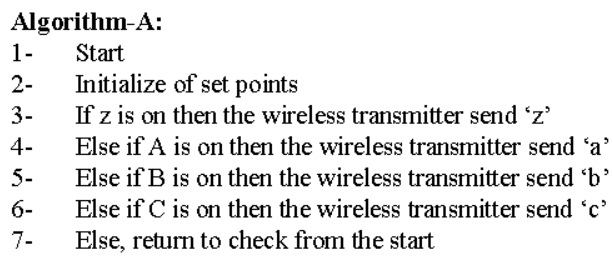

Algorithm-B:

1- Start

2- Initialize values of dimensions of $\mathrm{a} 1, \mathrm{a} 2 . \mathrm{b} 1, \mathrm{~b} 2$ and $\mathrm{c}$. Then the system check the received data $(x)$ and do the following instructions

3- If $x=$ ' $z$ ' and $j=0$ then the robot do no thing

4- Else if $x=$ ' $z$ ' and $j=1$ then the robot come back to $Z$ location achieve the distances a1 and $a 2$

5- Else if $x=' z$ ' and $j=2$ then the robot come back to $Z$ location achieve the distances $b 1$ and $b 2$

6- Else if $x=$ ' $z$ ' and $j=3$ then the robot come back to $Z$ location achieve the distance $c$

7- Else if $x=' a$ ' and $j=0$ then the robot moves to A location achieve the distances a1 and $\mathrm{a} 2$

8- Else if $x=$ ' $a$ ' and $j=1$ or $j=2$ or $j=3$ then the robot do no thing

9- Eels if $x=$ ' $b$ ' and $j=0$ then the robot moves to $B$ location achieve the distances $b 1$ and $b 2$

10- Else if $x=' b$ ' and $j=1$ or $j=2$ or $j=3$ then the robot do no thing

11- Else if $x=' c$ ' and $j=0$ then the robot moves to $C$ location achieve the distance $c$

12- Eels if $x={ }^{\prime} c{ }^{\prime}$ and $j=1$ or $j=2$ or $j=3$ then the robot do no thing

\section{CONCLUSION}

This project offers a mobile robot controlled wirelessly through a PC-control unit this robot can be used in many fields as industrial applications, domestic services, hazardous fields, agriculture and 
medical applications. In this system, the wireless technique eliminates the need to the flexible wires in the work field of the mobile robot and its reduces costs of the installation and maintenance. The proposed robot can travel in the forward and the backward directions and its can rotate to the left and the right rotation, also it can stop these directions of the robot is wirelessly controlled by the costumer through the designed GUI in the PC control unit. This robot is tested and its achieved very high accuracy in the navigating for the determined paths. This wirelessly controlled mobile robot reaches many advantages as, its need low power; it has the simplicity and flexibility for using it by the costumer.

\section{REFERENCES}

Borker, K., R. Gaikwad and A. Rajput, 2014. Wireless controlled surveillance robot. Intl. J. Adv. Res. Comput. Sci. Manage. Stud., 2: 436-441.

Chen, W., Y. Zhao, C. Liu, M. Jiang and J. Sun, 2017. Design and implementation of control system for nuclear pollution disposal robot based on wireless communication. Proceedings of the 2017 2nd Conference on Advanced Information Technology, Electronic and Automation Control (IAEAC), March 25-26, 2017, IEEE, Chongqing, China, ISBN:978-1-4673-8980-8, pp: 2475-2478.

Farkhana, M. and A.A. Hanan, 2008. Mobility in mobile ad-hoc network testbed using robot: Technical and critical review. Rob. Auton. Syst., 108: 153-178.
Kahar, S., R. Sulaiman, A.S. Prabuwono, N.A. Ahmad and M.A.A. Hassan, 2012. A review of wireless technology usage for mobile robot controller. Proceedings of the 2012 International Conference on System Engineering and Modeling (ICSEM 2012) Vol. 34, April 1, 2012, IACSIT Press, Singapore, pp: 7-12.

Lee, H.T., H.L. Tsai, Z.Q. Chen and Y.T. Jiang, 2014. Mobile detecting robot controlled by Smartphone based on IOS. Eng., 6: 750-757.

Li, H., B. Li and W. Xu, 2015. Development of a remote-controlled mobile robot with binocular vision for environment monitoring. Proceedings of the 2015 International Conference on Information and Automation, August 8-10, 2015, EEE, Lijiang, China, ISBN:978-1-4673-9103-0, pp: 737-742.

Patil, A.D., H.I. Kadiri, A.S. Joshi and A.B. Wani, 2016. IOT based remote access human control robot using MEMS sensor. Intl. J. Sci. Mobile Comput., 5: 816-826.

Teodorescu, R.M., I.B. Cioc, B.A. Vochin and A.I. Lita, 2016. LabVIEW application used for remote control of a mobile robot with XBEE communication. Proceedings of the 2016 8th International Conference on Electronics, Computers and Artificial Intelligence (ECAI), June 30-July 02, 2016, IEEE, Ploiesti, Romania, ISBN:978-1-5090-2048-5, pp: 1-4. 